\title{
CONTROLAR LÍQUIDOS: UMA INTERVENÇÃO DE ENFERMAGEM PARA O PACIENTE COM EXCESSO DE VOLUME DE LÍQUIDOS ${ }^{1}$
}

\author{
Heloísa Cristina Quatrini Carvalho Passos Guimarães ${ }^{2}$
} Alba Lúcia Botura Leite de Barros ${ }^{3}$

Guimarães HCQCP, Barros ALBL. Controlar líquidos: uma intervenção de enfermagem para o paciente com excesso de volume de líquidos. Rev Latino-am Enfermagem 2003 novembro-dezembro; 11(6):734-41.

Objetivou-se, com este estudo, identificar quais das atividades de enfermagem, contidas na intervenção Controle de líquidos, proposta pela Classificação das Intervenções de Enfermagem (Nursing Intervention Classification-NIC), são realizadas e consideradas importantes por enfermeiras, para o atendimento de pacientes com o diagnóstico de enfermagem Excesso de volume de líquidos, proposto pela North American Nursing Diagnoses Association (NANDA). Os dados foram coletados em três hospitais. A amostra constou de 77 enfermeiros, que escolheram a melhor alternativa em uma escala de Likert, para indicar quais dessas atividades consideravam importantes realizar e quais eram realizadas na sua prática diária assistencial. Concluiuse que a maioria das atividades de enfermagem foram consideradas sempre importantes e sempre realizadas pelas enfermeiras. Essas atividades foram classificadas como independentes e dependentes.

DESCRITORES: cuidados de enfermagem; processos de enfermagem; diagnóstico de enfermagem

\section{FLUID MANAGEMENT: A NURSING INTERVENTION FOR THE PATIENT WITH FLUID VOLUME EXCESS}

The objective of this research is to identify which nursing activities contained in the fluid management intervention proposed by the Nursing Intervention Classification (NIC) are realized and considered important by nurses, to attend patients who received fluid volume excess as a nursing diagnosis, as proposed by the North American Nursing Diagnoses Association (NANDA). Data were collected in three hospitals. The sample included 77 nurses, who chose the best alternative on a Likert scale, to indicate the realization of which of those actives they considered important and which activities are realized in their daily care practice. It was concluded that most of the nursing activities were always considered important and were always performed by the nurses. These activities were classified as independent and dependent.

DESCRIPTORS: nursing care; nursing process; nursing diagnosis

\section{CONTROLAR LÍQUIDOS: UNA INTERVENCIÓN DE ENFERMERÍA PARA EL PACIENTE CON EXCESO DE VOLUMEN DE LÍQUIDOS}

El objetivo de este estudio fue identificar cuales de las actividades de la enfermería contenidas en la intervención de Control de los líquidos propuesta por la clasificación de las intervenciones de la enfermería (Nursing Intervention Classification - NIC), son realizadas y consideradas importantes por las enfermeras, para el cuidado de pacientes con diagnóstico de enfermería de exceso del volumen de líquidos propuesto por la Norht American Nursing Diagnoses Associaton. Los datos fueron recolectados en tres hospitales, y la muestra constó de 77 enfermeros que escogieron la mejor alternativa en una escala de Likert, para indicar cuales de estas actividades consideraban importantes de realizar y cuales eran realizadas en su práctica asistencial. Se concluyó que la mayoría de las actividades de la enfermería fueron siempre consideradas importantes y siempre realizadas por las enfermeras. Estas actividades fueron clasificadas como: independientes y dependientes.

DESCRIPTORES: atención de enfermería; procesos de enfermería; diagnóstico de enfermería

\footnotetext{
${ }^{1}$ Trabalho extraído da Tese de Doutorado "Intervenções de Enfermagem Propostas pela Nursing Intervention Classification (NIC) para o diagnóstico de enfermagem de excesso de volume de líquidos” apresentada ao Programa de Pós-Graduação em enfermagem, UNIFESP/EPM, 2000, apresentado na $14^{\text {th }}$ Biennial Conference on Nursing Diagnosis-NANDA: Forging LINKS to the Future. Orlando, Florida, 2000, vinculado ao Projeto de Pesquisa do CNPq "Sistematização de Assistência de Enfermagem em hospitais de ensino", n.521359/97-0; ${ }^{2}$ Enfermeira, Doutor em Enfermagem, Pesquisador Científico III do Instituto Lauro de Souza Lima, e-mail: heloisaquatrini@ig.com.br; ${ }^{3}$ Enfermeira, Doutor em Ciências pela Escola Paulista de Medicina, Professor Adjunto, Diretora de Enfermagem do Hospital São Paulo, da Universidade Federal de São Paulo
} 
INTRODUÇÃO

$\boldsymbol{A}$ enfermagem atua no controle de líquidos dos pacientes com excesso de volume de líquidos (EVL), embora utilize diversas terminologias para descrever $\mathrm{O}$ mesmo resultado, prejudicando, às vezes, o entendimento e a comunicação dessas intervenções. Para que a enfermagem utilize linguagem uniforme se faz premente a padronização da nomenclatura em uso. A utilização de classificações para diagnósticos, intervenções e resultados de enfermagem facilita tanto a uniformização da prática como da linguagem específica. Neste estudo, foram utilizadas as classificações de diagnósticos North American Nursing Diagnoses Association (NANDA) ${ }^{(1)}$, e de intervenções de enfermagem Nursing Intervention Classification $(\mathrm{NIC})^{(2)}$, pois ambas estão no projeto da Classificação Internacional da Prática de Enfermagem (CIPE), um esforço do Conselho Internacional de Enfermeiros para unificar a prática profissional. A NIC propõe 19 intervenções, todas compostas por uma lista de atividades, para o diagnóstico de enfermagem excesso de volume de líquidos. Destaca-se como principais: controlar e monitorizar líquidos. A nossa proposta é investigar a intervenção controlar líquidos, definida como a "promoção do balanço de líquidos e a prevenção de complicações resultantes de níveis desejáveis ou não de líquidos"(2).

Embora não utilizando uma Classificação de intervenção de Enfermagem muitos autores propõem tomadas de decisões para o enfermeiro intervir diante do paciente que apresenta os fatores relacionados e as características definidoras do diagnóstico de enfermagem de excesso de volume de líquidos ${ }^{(3-5)}$.

$\mathrm{Na}$ área médica também há muitas referências para o embasamento teórico no caso de falência de bomba cardíaca, como o mecanismo de controle da insuficiência cardíaca congestiva (ICC). Para a melhoria do desempenho da bomba cardíaca são utilizados glicosídeos digitálicos, agentes simpatominéticos, inotrópicos positivos, marcapasso; na redução do trabalho cardíaco é indicado repouso físico e emocional, tratamento da obesidade, terapêutica vasodilatadora, circulação assistida; e no controle do excesso de sal e da retenção hídrica, a dieta hipossódica, diuréticos, remoção mecânica de fluido com cateteres ${ }^{(6)}$.

Outro referencial importante é sobre edema pulmonar que está quase sempre associado à insuficiência cardíaca aguda ou crônica. A falência cardíaca, por sua vez, decorre da isquemia miocárdica aguda (com ou sem infarto prévio), hipertensão arterial, doença valvar, doença miocárdica primária e cardiopatia congênita. A insuficiência cardíaca pode ou não ser precipitada por taquiarritmias. O diagnóstico de edema agudo de pulmão é clínico e suas manifestações são dependentes do grau de líquidos acumulados, sendo o tratamento o seguinte: administração de oxigênio, diuréticos, elevação do decúbito, oxigênio úmido através de máscara facial, com efeito, venturi, correção da hipoxemia, opiáceos, como morfina (ajudam a reduzir a pré-carga e os reflexos pulmonares responsáveis pela dispnéia), entubação orotraqueal no momento exato, ventilação mecânica pode ser usada junto com pressão inspiratória positiva final ${ }^{(7)}$.

Nos dois parágrafos anteriores encontramos atividades dependentes e independentes da enfermagem ${ }^{(2)}$, e consideramos, mais uma vez, a importância do estabelecimento da competência do enfermeiro.

O diagnóstico de enfermagem excesso de volume de líquidos é um dos mais freqüentes em cardiopatas. Isso nos leva a questionar quais intervenções de enfermagem estão sendo realizadas em benefício desses pacientes $^{(8)}$.

\section{OBJETIVO}

- identificar quais atividades, dentre aquelas contidas na intervenção controle de líquidos, proposta pela NIC, para o diagnóstico de enfermagem excesso de volume de líquidos, são realizadas e consideradas importantes por enfermeiras, para o atendimento de pacientes cardiopatas que possuem esse diagnóstico.

\section{MÉTODO}

O estudo foi realizado em três hospitais da cidade de São Paulo, sendo dois especializados em cardiologia (aqui denominados Hospital A e Hospital B) e um hospital geral, que conta com unidades de Cardiologia, de Cirurgia Cardiovascular e de Pós-operatório de Cirurgia Cardíaca (Hospital C).

Os critérios para inclusão neste estudo foram: ser enfermeiras, trabalhar na assistência a cardiopatas há pelo menos dois anos e aceitar participar da pesquisa, dando 
seu consentimento por escrito. Seguindo esses parâmetros, obtivemos uma amostra de 77 enfermeiras, assim distribuídas: 36 no Hospital A, 23 no Hospital B e 18 no Hospital C.

Para a coleta de dados elaboramos dois instrumentos que continham apenas a lista e definição das atividades de enfermagem, proposta pela NIC, para intervenção Controlar Líquidos. Essa lista de atividades e sua definição foram previamente adequadas semanticamente ${ }^{(8-9)}$

Foram adotados para ambos os questionários 0 mesmo delineamento, alterando apenas o propósito de cada um. Desse modo, enquanto o primeiro levantava quais atividades eram consideradas importantes, o segundo detectava quais atividades eram executadas na prática diária. Como recurso para visualizar a opinião das enfermeiras em relação às atividades propostas pela NIC para a intervenção controle de líquidos, associamos uma escala intervalar do tipo Likert, para que essas registrassem, dentre as opções nunca (0), raramente (1), algumas vezes (2), muitas vezes (3), sempre (4), aquela que melhor indicasse seu parecer sobre a importância e 0 fazer profissional de cada atividade. Os instrumentos foram entregues às enfermeiras depois de orientadas quanto aos objetivos da pesquisa e obtidos seus consentimentos formais para participação na mesma. Nessa ocasião, foram preenchidos os questionários de identificação e também realizado esclarecimentos necessários sobre os instrumentos.

O pré-teste dos instrumentos foi realizado com quatro residentes de enfermagem de duas instituições da amostra. Como resultado, obteve-se a sugestão de acrescentar, na escala intervalar, a opção não se aplica (9), visto que algumas das atividades não se aplicavam ao contexto diário das enfermeiras. O teste permitiu estimar em trinta minutos a média de tempo gasto para responder os questionários. Outra acertiva inferida, após o pré-teste, foi que enfermeiras com menos de dois anos de experiência na área de cardiologia sentiram-se inseguras ao responder o questionário. Isso levou a estabelecer, como critério de inclusão na amostra, a necessidade de experiência na área igual ou maior que dois anos.

A coleta de dados realizou-se após a aprovação dos projetos pelos Comitês de Ética em Pesquisa dos Hospitais A e C, e ciência da Diretora de Enfermagem, no Hospital B, que não contava com Comitê de Ética em Pesquisa.

A estratégia metodológica para analisar os resultados foi a de construir um plano cartesiano, tomando como coordenadas as dimensões fazer (realiza na prática diária) e importância (considerada importante realizar).

Após a aplicação dos questionários, foram feitas as médias entre as respostas (nunca $=0$ e sempre $=4$ ) de cada atividade, que estão representadas pelos números das atividades no plano cartesiano na intervenção controle de líquidos como uma das principais para o diagnóstico excesso de volume de líquidos. Dessa forma, padrões de concordância e discordância entre fazer e importância puderam ser analisados graficamente.

Nessa etapa, com a finalidade de destacar padrões finos de distinção, foi realizada a análise de agrupamento (Técnica de Cluster) ${ }^{(10)}$, onde é realizada análise para verificar a possibilidade de existência de classificações, e foi utilizado o recurso do zoom, para facilitar a identificação dos grupos (Grupo 1, 2, 3 e 4).

Analisando a intervenção Controlar líquidos para o diagnóstico de excesso de volume de líquidos, percebemos uma abrangência no que se refere à tomada de decisão da enfermeira. Essa intervenção contém atividades dependentes e independentes. As "atividades dependentes" são aquelas originadas de ordens médicas e as "atividades independentes" representam as que correspondem a iniciativas exclusivas da enfermeira ${ }^{(2)}$. Investigamos a análise das concordâncias perfeitas dessas atividades através do Teste de Wilcoxon ${ }^{(11)}$.

O julgamento a respeito dos grupos de atividades foi baseado na experiência clínica das autoras e no conhecimento a respeito da sua execução, dependendo ou não de ordens médicas. Sendo assim, algumas dessas atividades foram classificadas como dependentes e independentes, de acordo com o que conhecemos sobre a rotina dos hospitais estudados e a experiência das enfermeiras que fizeram parte da amostra.

Para facilitar o entendimento do que representa cada número presente nas Figuras 1 e 2, inserimos a legenda abaixo indicada.

- 1) Pesar diariamente e monitorar tendências.

- 2) Contar ou pesar fraldas de forma apropriada.

- 3) Manter registro acurado de ingesta e débito de líquidos.

- 4) Inserir cateter urinário, se apropriado.

- 5) Monitorizar estado de hidratação (por exemplo: membranas mucosas úmidas, adequação dos pulsos e pressão sangüínea ortostática), como apropriada.

- 6) Monitorizar resultados laboratoriais relevantes para retenção de líquidos (por exemplo: densidade urinária 
aumentada, hematócrito diminuído e níveis de osmolalidade urinária aumentado).

- 7) Monitorizar estado hemodinâmico, incluindo PVC, PAM, PAP, PCP, se disponíveis.

- 8) Monitorizar sinais vitais, como apropriado.

- 9) Monitorizar indicações de sobrecarga/retenção de líquidos (por exemplo: estertores crepitantes, PVC ou PCP elevadas, edema, distensão das veias do pescoço e ascite), como apropriado.

- 10) Monitorizar mudança de peso antes e após diálise, se apropriado.

- 11) Avaliar localização e extensão do edema, se presente.

- 12) Monitorizar alimentos/líquidos ingeridos e calcular aporte calórico diário, como apropriado.

- 13) Administrar terapia endovenosa, como prescrita.

- 14) Monitorizar estado nutricional.

- 15) Dar líquidos, como apropriado.

- 16) Administrar diuréticos prescritos, como apropriado.

- 17) Administrar líquidos por via endovenosa em temperatura ambiente.

- 18) Promover ingestão oral (por exemplo: providenciar um canudo, oferecer líquidos entre as refeições e trocar água rotineiramente), como apropriado.

- 19) Instruir o paciente sobre jejum oral, como apropriado.

- 20) Distribuir a ingesta de líquidos nas 24 horas, como apropriado.

- 21) Encorajar o acompanhante/cuidador a auxiliar o paciente com a alimentação, como apropriado.

- 22) Oferecer lanches (bebidas freqüentes e frutas frescas/ sucos de frutas), como apropriado.

- 23) Restringir ingesta de água livre na presença de hiponatremia dilucional, com níveis de sódio sérico abaixo de $130 \mathrm{mEq}$ por litro.

- 24) Monitorizar resposta do paciente à terapia eletrolítica.

- 25) Consultar médico se os sinais/sintomas de excesso de volume de líquidos persistirem ou piorarem.

- 26) Providenciar disponibilidade de produtos sangüíneos para transfusão, se necessário.

- 27) Preparar para administração de produtos sangüíneos (checar sangue com a identificação do paciente e preparar o material para infusão), se necessário.

- 28) Administrar produtos sangüíneos (por exemplo: plaquetas e plasma fresco congelado), como apropriado.

\section{RESULTADOS E DISCUSSÃO}

Ao examinarmos as atividades contidas na intervenção controle de líquidos, comparando-as com as atividades realizadas na prática clínica e a importância atribuída às mesmas pelas enfermeiras entrevistadas, verificamos que todas as atividades foram apontadas como sempre ou muitas vezes realizadas, o mesmo ocorrendo com a variável importância (Figura 1).

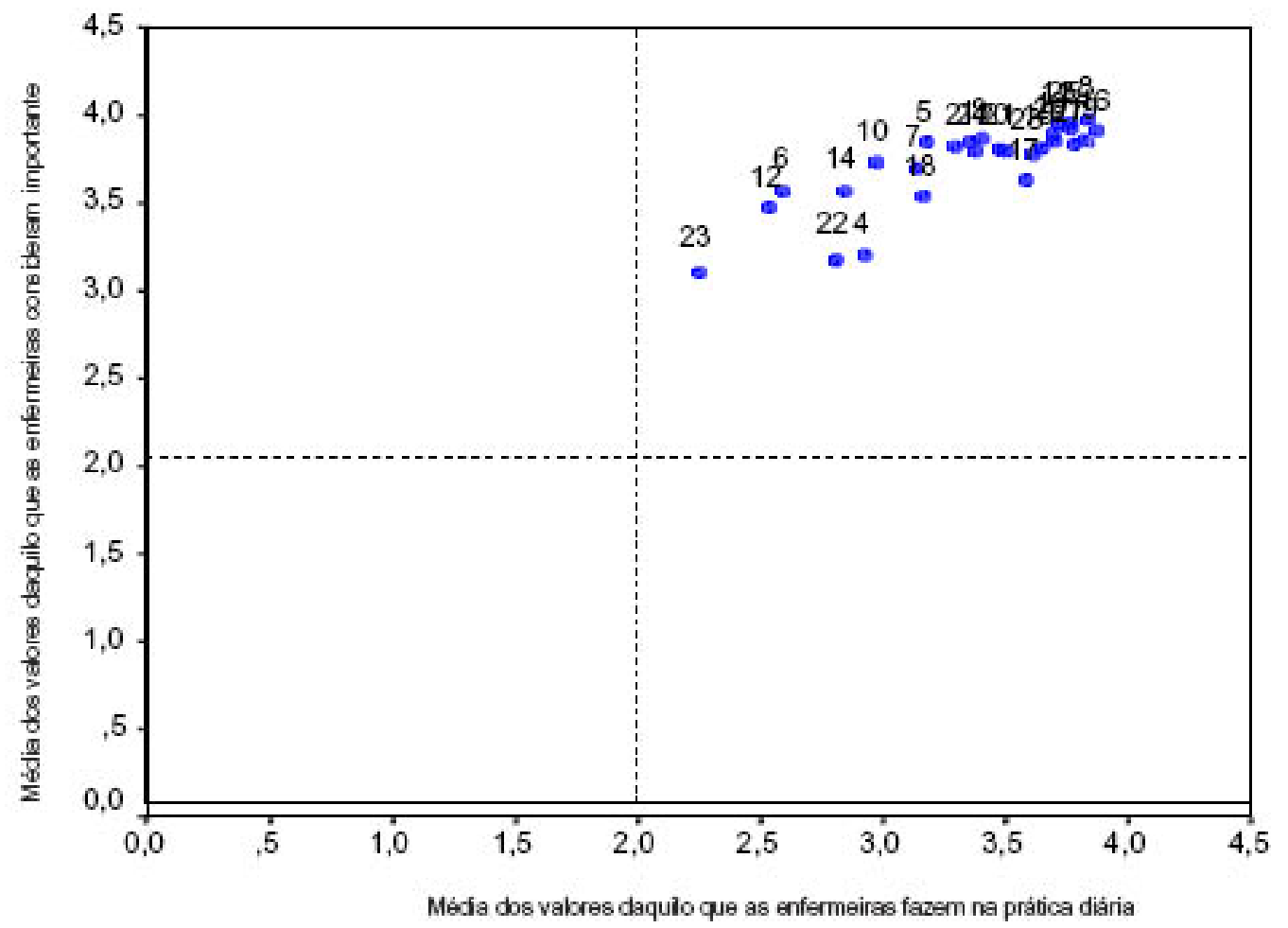

Figura 1 - Distribuiçảo (em clusters) dos valores médios das respostas entre 77 enfermeiras, das atividades de enfermagem da intervençăo controle de líquidos da NIC, segundo a sua realizaçäo e importância. Säo Paulo, 2000 
Ao observarmos esse aglomerado de atividades (o cluster), que à primeira vista parece confuso e de difícil entendimento, sentimos a necessidade de utilizarmos o recurso do zoom (aproximação), e verificamos que as atividades aglomeradas (representadas, na figura, por números) no plano de fazer e no plano importância se concentraram no quadrante superior esquerdo com o predomínio das respostas sempre (4) e muitas vezes (3), entretanto, apresentavam discretas diferenças, sugerindo a possibilidade de existir uma classificação das atividades dentro desse quadrante (Figura 2).

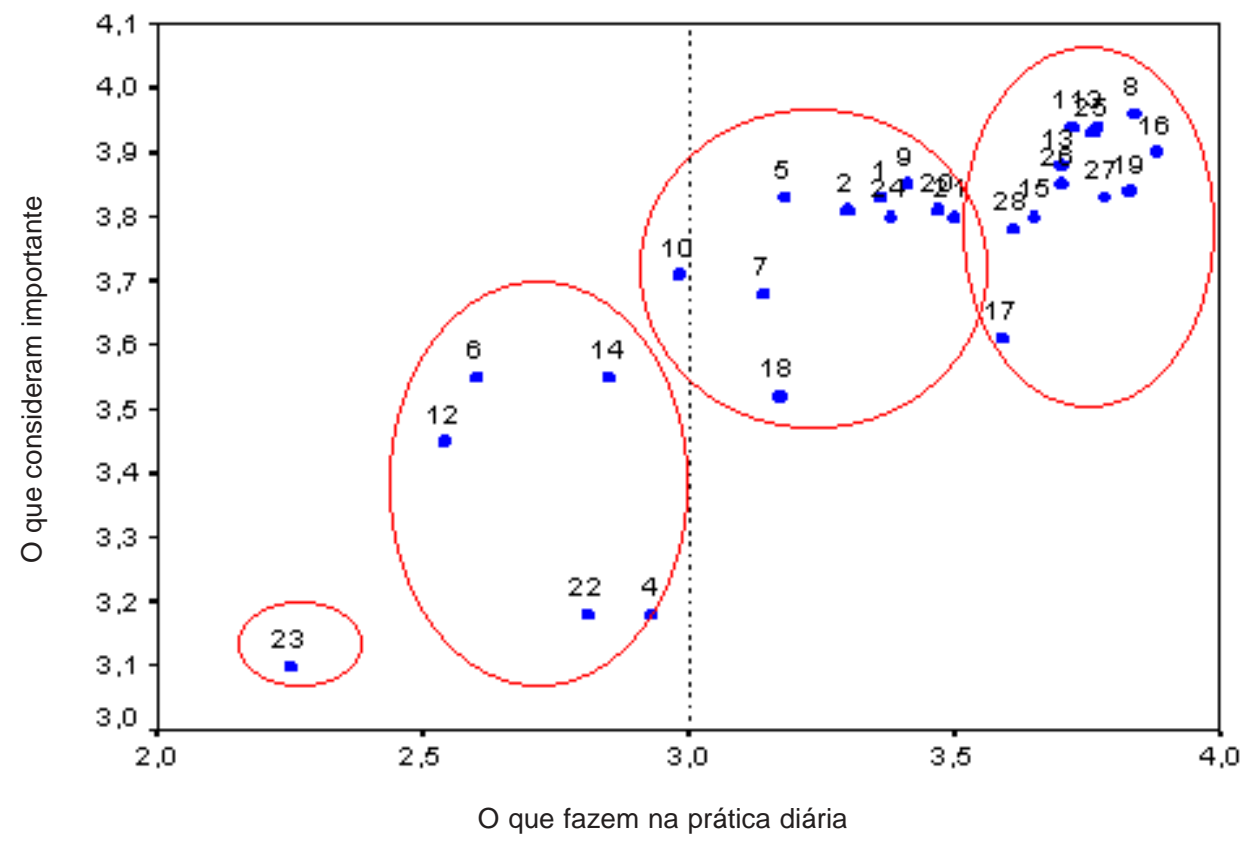

Figura 2 - Distribuição em cluster, com aproximação (zoom), das médias dos valores das respostas entre as 77 enfermeiras, das atividades de enfermagem da intervenção controle de líquidos da NIC, segundo a sua realização e importância. São Paulo, 2000

A análise pela Técnica de Cluster sugeriu a existência de 4 grupos de atividades (Figura 2), que foram denominados de G1, G2, G3 e G4. Cada um desses grupos mostra as atividades divididas em "dependentes" e "independentes". Chamamos a atenção para o fato de que algumas atividades podem ser consideradas tanto independentes como dependentes.

Verificamos que os 4 grupos (clusters) formados têm diferentes composições das atividades, contidas na intervenção controlar líquidos, no que diz respeito à dependência e independência.

Tabela 1 - Grupo de atividades 1 Dependentes $\left(D^{\star}\right)$ e Independentes $\left(I^{\star *}\right)$ da Intervenção Controlar Líquidos da NIC. São Paulo, 2000

\begin{tabular}{lcc}
\multicolumn{1}{c}{ Grupo de Atividades - G1 } & $\mathrm{D}^{*}$ & $\mathrm{I}^{* *}$ \\
3) Manter registro acurado de ingesta e débito de líquidos & $X$ & $\mathrm{X}$ \\
8) Monitorizar sinais vitais, como apropriado & $X$ & $\mathrm{X}$ \\
11) Avaliar localização e extensão do edema & $\mathrm{X}$ & $\mathrm{X}$ \\
13) Administrar terapia endovenosa, como prescrito & $\mathrm{X}$ \\
15) Dar líquidos como apropriado & $\mathrm{X}$ \\
16) Administrar diuréticos, como prescrito & $\mathrm{X}$ \\
17) Administrar líquidos por via endovenosa & $\mathrm{X}$ \\
19) Instruir paciente sobre jejum oral & \\
25) Consultar médico se sinais e sintomas de excesso de volume de líquidos & $\mathrm{X}$ \\
persistirem & $\mathrm{X}$ \\
26) Providenciar disponibilidade de produtos sangüíneos para transfusão, se & \\
necessário & \\
27) Preparar administração de produtos sangüíneos, como apropriado & $\mathrm{X}$ \\
\hline
\end{tabular}

A numeração apresentada na Tabela 1 e subseqüentes refere-se à ordem na qual as atividades propostas pela NIC aparecem na intervenção controlar líquidos ${ }^{2}$. 
Foi realizado um estudo de validação de conteúdo ${ }^{(12)}$ sobre 15 intervenções relacionadas ao balanço de líquidos e de eletrólitos. Cada intervenção foi acompanhada de sua definição e atividades. Nesse, a intervenção controle de líquidos atingiu o score de 0,77, estabelecendo as atividades principais e de suporte ${ }^{(13)}$.

As 7 atividades negritadas no grupo 1 correspondem às atividades verificadas, neste estudo, como as mais realizadas e consideradas mais importantes e detectadas como coincidentes com as atividades críticas ou principais, encontradas no estudo citado. Destaca-se ainda outras atividades como principais, quais sejam: "dar líquidos, como apropriado; instruir o paciente sobre jejum oral e monitorar resultados laboratoriais relevantes à restrição hídrica"(13). Constatamos que administração de diuréticos prescritos foi uma atividade também referida por vários autores $^{(3-7)}$.

Assim, entre as atividades dependentes, as enfermeiras, neste estudo, referiram realizar e considerar importantes as seguintes: 13) administrar terapia endovenosa como prescrito; 16) administrar diuréticos como prescrito. Tais atividades correspondem a condutas médicas para Insuficiência Cardíaca Congestiva, sendo as duas primeiras muito realizadas e consideradas muito importantes, de acordo com resultados apresentados na Figura 2, e fazem parte, portanto, do grupo de atividades dependentes (grupo 1).

A partir das considerações dos livros de texto de enfermagem, verificamos que a atividade 3) manter registro acurado de ingesta e débito de líquidos, é uma proposta de tratamento de enfermagem ${ }^{(3-5,13)}$. Outras atividades propostas são: 8) monitorizar sinais vitais, como apropriado e 11) avaliar localização e extensão do edema $a^{(3-5,14-15)}$. As atividades 13) administrar terapia endovenosa como prescrito, 15) dar líquidos como apropriado, 17) administrar líquidos por via endovenosa também são consideradas parte do tratamento de enfermagem ${ }^{(5)}$.

Quanto às atividades 19) instruir paciente sobre jejum oral, 25) consultar médico se sinais e sintomas de excesso de volume de líquidos persistirem, 26) providenciar disponibilidade de produtos sangüíneos para transfusão, se necessário e 27) preparar a administração de produtos sangüíneos foram encontradas nos resultados deste estudo (nos 3 hospitais), sendo consideradas como muito realizadas e muito importantes e, como vemos na Figura 2, pertencem ao grupo 1, ou seja, são dependentes. Entretanto, não foram descritas nos livros de texto de enfermagem e medicina. As atividades 16) administrar diuréticos, como prescrito e 17) administrar terapia endovenosa, como prescrito foram encontradas em livros de texto médicos e de enfermagem.

Tabela 2 - Grupo de atividades 2 Dependentes ( $\left.D^{*}\right)$ e Independentes $\left(I^{\star \star}\right)$ da Intervenção Controlar Líquidos da NIC. São Paulo, 2000

\begin{tabular}{|c|c|c|}
\hline Grupo de Atividades - G2 & $\mathrm{D}$ & $\begin{array}{l}\mathrm{y} \\
\mathrm{y}\end{array}$ \\
\hline 1) Pesar diariamente e monitorar tendências & $\mathrm{X}$ & $X$ \\
\hline 2) Contar e pesar fraldas & $\mathrm{X}$ & $X$ \\
\hline $\begin{array}{l}\text { 5) Monitorizar estado de hidratação } \\
\text { 7) Monitorizar estado hemodinâmico } \\
\text { 9) Monitorizar indicações de sobrecarga/retenção de } \\
\text { líquidos, como apropriado }\end{array}$ & $\mathrm{X}$ & \\
\hline $\begin{array}{l}\text { 10) Monitorizar mudança de peso antes e após diálise, } \\
\text { se apropriado }\end{array}$ & $\mathrm{X}$ & \\
\hline $\begin{array}{l}\text { 18) Promover ingestão oral (por exemplo: providenciar } \\
\text { canudo) oferecer líquidos entre as refeições e trocar } \\
\text { água rotineiramente }\end{array}$ & & \\
\hline ouir ingesta de líquidos nas 24 horas & & \\
\hline $\begin{array}{l}\text { 21) Encorajar o acompanhante e cuidador a auxiliar o } \\
\text { paciente na alimentação, como apropriado }\end{array}$ & & \\
\hline $\begin{array}{l}\text { 24) Monitorizar resposta do paciente à terapia } \\
\text { eletrolítica }\end{array}$ & K & \\
\hline
\end{tabular}

A atividade monitorar peso antes e após diálise, encontrada na literatura médica ${ }^{(6-7)}$, faz parte do grupo 2 , no qual predominaram atividades consideradas independentes.

Existe trabalho constatando que $97,5 \%$ das enfermeiras prescreveram pesar em jejum para pacientes portadores de excesso de volume de líquidos ${ }^{(8)}$. Sabemos que o peso é o indicador mais sensível do equilíbrio hídrico, assim o ganho de peso abrupto em pacientes com falência cardíaca é sinal de retenção de líquidos, por isso a pesagem diária está recomendada para os pacientes com características definidoras e fatores relacionados ao excesso de volume de líquidos ${ }^{(3-5,14)}$. Acreditamos também ter sido, por isso, considerada como atividade principal no estudo citado anteriormente ${ }^{(13)}$.

Tabela 3 - Grupo de atividades 3 Dependentes $\left(D^{*}\right)$ e Independentes $\left(I^{\star \star}\right)$ da Intervenção Controlar Líquidos da NIC. São Paulo, 2000

\begin{tabular}{llll}
\hline \multicolumn{1}{c}{ Grupo de Atividades - G3 } & D & I \\
4) Inserir cateter urinário, se apropriado & $X$ & $X$ \\
6) Monitorizar resultados laboratoriais relevantes para & $X$ & \\
retenção de líquidos (por exemplo: densidade urinária & & \\
aumentada, hematócrito diminuído e níveis de & & \\
osmolalidade urinária aumentado) & & & \\
12) Monitorizar alimentos/líquidos ingeridos e calcular & $X$ & $X$ \\
aporte calórico & & & $X$ \\
14) Monitorizar estado nutricional, como apropriado & $X$ & $X$ \\
\hline
\end{tabular}


A proporção das atividades dependentes e independentes no grupo 3 é de $50 \%$. Ao avaliarmos as atividades que pertencem a esse grupo, percebemos que a atividade 4) inserir cateter urinário, faz parte da rotina de enfermagem para algumas enfermeiras, sendo considerada atividade independente, outras enfermeiras só a realizam com o pedido médico, tornando-a atividade dependente. A atividade 6) monitorizar resultados laboratoriais relevantes para a retenção de líquidos, ainda não foi incorporada na avaliação clínica pelas enfermeiras brasileiras. As atividades 12) monitorizar alimentos líquidos ingeridos e calcular aporte calórico e a 14) monitorizar estado nutricional estão relacionadas com a nutrição do paciente, a enfermeira pode contribuir avaliando o estado nutricional do mesmo e observando manifestações clínicas de carências nutricionais sabendo que a estrutura de um corpo, sadio ou não, é constituída por substâncias oriundas dos alimentos, assimiladas e transformadas por esse organismo e que nenhum fenômeno orgânico normal ou anormal ocorre sem o envolvimento de um componente nutricional. Quando a alimentação do indivíduo não é quanti-qualitativamente suficiente, o indivíduo entra em processo de desnutrição, o que diminui sua competência imunológica, retarda a cicatrização, favorece o aparecimento de úlceras de decúbito e leva à insuficiência de múltiplos órgãos ${ }^{(16)}$.

Tabela 4 - Grupo de atividades 4 Dependentes $\left(D^{*}\right)$ e Independentes $\left(I^{\star \star}\right)$ da Intervenção Controlar Líquidos da NIC. São Paulo, 2000

\begin{tabular}{lll}
\hline Grupo de Atividades - G4 & $\mathrm{D}$ & $\mathrm{I}$ \\
23) Restringir ingesta de água livre na & $\mathrm{X}$ & \\
presença de hiponatremia dilucional com & \\
níveis de sódio sérico abaixo de $130 \mathrm{mEq} / \mathrm{l}$ & \\
\hline
\end{tabular}

A atividade do grupo 4, apesar de apontada como sempre realizada e sempre considerada importante não faz parte da rotina de enfermagem, nem é descrita como atividade própria a essa situação clínica ${ }^{(3-5,15)}$.

Tabela 5 - Classificação das seis atividades consideradas iguais, pelo Teste de Wilcoxon, quanto ao tipo de atividade e ao grupo ao qual pertencem. São Paulo, 2000

\begin{tabular}{|c|c|c|c|c|c|c|c|c|}
\hline Atividades & \multicolumn{4}{|c|}{ Dependentes } & \multicolumn{4}{|c|}{ Independentes } \\
\hline & G1 & G2 & G3 & G4 & G1 & $\mathrm{G} 2$ & G3 & G4 \\
\hline 4)Inserir cateter urinário & & & $\mathrm{X}$ & & & & $\mathrm{x}$ & \\
\hline $\begin{array}{l}\text { 15)Dar líquidos, como } \\
\text { apropriado }\end{array}$ & $X$ & & & & & & & \\
\hline $\begin{array}{l}\text { 17)Administrar líquidos } \\
\text { por EV }\end{array}$ & $\mathrm{X}$ & & & & & & & \\
\hline $\begin{array}{l}\text { 19) Instruir paciente sobre } \\
\text { jejum oral }\end{array}$ & $X$ & & & & & & & \\
\hline $\begin{array}{l}\text { 27)Preparar } \\
\text { administração de sangue }\end{array}$ & $\mathrm{X}$ & & & & $\mathrm{X}$ & & & \\
\hline $\begin{array}{l}\text { 16)Administrar diuréticos } \\
\text { prescritos }\end{array}$ & $x$ & & & & & & & \\
\hline
\end{tabular}

A análise de medidas precisas de concordância, através do Teste de Wilcoxon, demonstrou que existem seis atividades que são perfeitamente iguais, no que diz respeito à sua realização pelas enfermeiras na prática clínica e à importância que lhes é atribuída pelas mesmas. Ao classificá-las de acordo com o tipo de atividade e o grupo a que pertence, obtivemos os dados da Tabela 5.

Das 6 ações, 5 pertencem ao grupo 1, ou seja, sempre e muitas vezes realizadas e consideradas importantes sempre e muitas vezes. Dessas 5 , segundo o referencial adotado, verificamos que apenas uma segue como independente e, ainda assim, parte da amostra a considera dependente. A sexta atividade 4) Inserir cateter urinário, que pertence ao grupo 3 , é considerada por algumas enfermeiras como dependente e por outras como independente, segundo a experiência clínica de cada uma.

\section{CONSIDERAÇÕES FINAIS}

Vale destacar que algumas enfermeiras, ao entrarem em contato com o nosso instrumento, com o título de intervenção da NIC, controle de líquidos, para o paciente com excesso de volume de líquidos, com suas definições e atividades, mostraram-se muito entusiasmadas dizendo: ...é isto que nós precisávamos...

Além disso, as poucas sugestões de novas intervenções realizadas pelas enfermeiras que fizeram parte da nossa amostra, na sua grande maioria, já faziam parte das atividades da NIC, mas escritas de maneira diferente, sendo, portanto, uma questão apenas de interpretação. Entre as atividades sugeridas pelas enfermeiras, uma delas tem relação com o respeito ao indivíduo: valorizar $\boldsymbol{e}$ respeitar, dentro de limites, a vontade do paciente (mencionada uma vez) que reflete a preocupação com o paciente como um todo. Outra sugestão foi monitorizar distúrbios de comportamento (citada uma vez), feita, provavelmente, devido às alterações metabólicas presentes nos pacientes com excesso de volume de líquidos.

Acreditamos que essas são sugestões importantes, apesar de pouco numerosas. Devemos considerar a possível inclusão da mesma nas intervenções propostas pela NIC para diagnóstico excesso de volume de líquidos.

Insistimos ainda que, para implementar a NIC, fazse necessário o estabelecimento do que é competência 
da enfermeira ou atividades independentes e do que é dependente de ordens médicas ou atividades dependentes, para garantir cuidados de qualidade e visibilidade das atividades da enfermeira.

\section{REFERÊNCIAS BIBLIOGRÁFICAS}

1. NANDA. Nursing diagnoses: definitions \& classification 1999-2000. Philadelphia: Artes Médicas do Sul; 2000.

2. McCloskey JC, Bulechek GM, editors. Nursing Interventions Classification (NIC). $3^{\text {th }}$ ed. St. Louis: Mosby - Year Book; 2000. 3. Smeltzer SC, Bare BG. Assistência a pacientes com distúrbios cardíacos In: Brunner LS, Suddarth DS. Tratado de Enfermagem Médico-Cirúrgica. $7^{\mathrm{a}}$ ed. Rio de Janeiro (RJ): Guanabara Koogan; 1994.

4. Hudak CM, Gallo BM. Cuidados intensivos de enfermagem: uma abordagem conceitual. $6^{\mathrm{a}}$ ed. Rio de Janeiro (RJ): Guanabara Koogan; 1996.

5. Black JM, Matassarin-Jacobs E. Luckmann \& Sorensen Enfermagem Médico-Cirúrgica: Uma abordagem psicofisiológica. $4^{\mathrm{a}}$ ed. Rio de Janeiro (RJ): Guanabara Koogan; 1996.

6. Braunwauld E. Tratado de medicina cardiovascular. $4^{\mathrm{a}} \mathrm{ed}$. São Paulo (SP): Roca; 1996.

7. Knobel E. Condutas no paciente grave. $2^{\mathrm{a}}$ ed. São Paulo (SP): Atheneu; 1998.

8. Guimarães HCQCP. Intervenções de enfermagem da Nursing Intervention Classification para paciente com diagnóstico de enfermagem de excesso de volume de líquidos. [tese]. São Paulo (SP): Universidade Federal de São Paulo/EPM; 2000.

9. Barros ALBL; Guimarães HCQCP. Semantic Adaptation of two Nursing Intervention Classification (NIC) proposed for the Fluid volume Excess. Conference 14 $14^{\text {th }}$ Biennial on Nursing Diagnosis- NANDA: Forging LINKS the Future. 2002 april 1013; Chicago; 2002.

10. Pereira JCR. Análise de dados qualitativos: estratégias de dados para as ciências da saúde, humanas e sociais. $2^{\text {a }}$ ed. São Paulo (SP): Editora Universidade São Paulo; 1999. 11. Siegel S. Estatística não paramétrica. São Paulo (SP): McGraw-Hill; 1975.

12. Fehring R. Methods to validate nursing diagnosis. Heart \& Lung 1987; 16(6):625-9.

13. Cullen L. Interventions related to fluid and electrolyte balance. Nurs Clin North Am 1992; 27(2):569-97.

14. Doenges ME, Moorhouse MF. Aplicação do processo de enfermagem. In: Diagnósticos e Intervenções em enfermagem. Porto Alegre (RS): Artes Médicas Sul; 1999. p. 18-21.

15. Carpenito LJ. Nursing care plans \& documentation: nursing diagnoses and colaborative problems. $3^{\underline{a}}$ ed. Pensylvania: Lippicott; 1997.

16. Valente FLS, Corso ACT. Implantação de sistema de vigilância alimentar e nutricional (SIVAN) junto aos pacientes internados no hospital universitário da UFSC. Saúde em Debate 1992; 36:81-92. 